\title{
BIOLOGICAL MARKERS AS INDICATORS OF PATHOLOGICAL RESPONSE TO PRIMARY CHEMOTHERAPY IN ORAL-CAVITY CANCERS
}

\author{
Aurora Costa $^{1 *}$, Lisa Licitra ${ }^{2}$, Silvia Veneroni ${ }^{1}$, Maria G. DAIDONE $^{1}$, Cesare Grandi ${ }^{3}$, Raffaele CAVINA ${ }^{2}$, Roberto Molinari $^{3}$ \\ and Rosella SILVESTRINI ${ }^{1}$ \\ ${ }^{1}$ Oncologia Sperimentale C, Istituto Nazionale per lo Studio e la Cura dei Tumori, Milan, Italy \\ ${ }^{2}$ Oncologia Medica C, Istituto Nazionale per lo Studio e la Cura dei Tumori, Milan, Italy \\ ${ }^{3}$ Oncologia Chirurgica Maxillo-Facciale, Istituto Nazionale per lo Studio e la Cura dei Tumori, Milan, Italy
}

The predictive role in terms of pathological response and prognostic role of biomarkers such as GST $=\pi$, p53, bcl-2 and bax expression, immuno-hist ochemically detected, and of the S-phase cell fraction, autoradiographically determined as thymidine labeling index (TLI), were investigated within a prospective randomized phase III clinical trial on squamouscell carcinoma of the oral cavity, including surgery or primary chem otherapy (PCT), which foresaw the prospective determination of biological markers. Pathological response was defined as the achievement after PCT of a pathological complete remission or the presence of microresidual disease. The study was performed on tum ors obtained from a series of 100 previously untreated patients with resectable T2-4N0-2M0 carcinoma. All biomarkers were unrelated, except for an inverse relation between TLI and GST - $\pi$ and a direct relation between bcl-2 and bax expression. In patients treated with surgery alone, 3-year disease-free survival (DFS) appeared to be weakly, but not significantly, related only to GST- $\pi$ and p53 expression. In patients treated with PCT, pathological response and DFS were independent of p53 expression and cell proliferation. Conversely, low GST $-\pi$ and bax expression were indicative of pathological response but lost relevance as predictors of DFS, whereas absence of bcl-2 was associated with high probability of 3-year DFS in the overall series as well as in non-responding patients. W ithin this latter sub-set, all patients with bcl-2-positive tumors relapsed within 1 year of surgery, whereas a $60 \%$ probability of 3-year DFS was observed for patients with bcl-2-negative tumors $(p=0.02)$. This interim analysis appears to indicate that some biofunctional markers can provide information on pathological response to PCT and could help in understanding treatment efficacy at a cellular level. Int. I Cancer (Pred. Oncol.) 79:619$623,1998$.

( 1998 Wiley-Liss, Inc.

Primary chemotherapy (PCT) for advanced head-and-neck cancer has been studied in clinical trials for more than a decade without a clear demonstration of benefit for local-regional tumor control or overall patient survival (Browman, 1994). The benefit derived from PCT's demonstrated ability to reduce distant failure is not matched by a clear benefit in terms of survival since it fails to improve local-regional control. PCT provides a high percentage of tumor response and, in some cases, ranging from $10 \%$ to $30 \%$, pathologically complete remission. Some patients may achieve a very good result, with the presence in the specimens of only microresidual disease. This means that a substantial fraction of tumors is highly chemosensitive to treatment and that patients may benefit from PCT. These important aspects for the cure of advanced head-andneck cancer patients should stimulate basic research to identify and evaluate biological variables in order to define a "biological profile" of a chemosensitive patient. Such variables could help clinicians to indicate the optimal treatment for an individual patient, thus maximizing treatment efficacy in terms of cure and organ preservation.

In the past, in vitro chemosensitivity assays have been proposed and used to define drug sensitivity on individual tumors at a pre-clinical level. However, the limited amount of tumor material before PCT and the moderate feasibility of in vitro tests represented, for this tumor type, important constraints on wide utilization of the approach. Conversely, the determination of biological markers involved or associated with different cellular aspects appears feasible on small tumor samples and has already provided predictive information on treatment response for several tumor types (Harris and Hollstein, 1993; Corvò et al., 1997; Silvestrini et al., 1998).

Molecular markers involved as determinants or targets of the cellular response to cytotoxic agents, including P-glycoprotein, glutathione $S$-transferase- $\pi$ (GST- $\pi$ ) and topoisomerases, were found to correlate with the efficacy of different pharmacological agents on clinical tumors. In particular, GST- $\pi$ has been reported to be indicative of cisplatin resistance (Okuyama et al., 1994), and GST- $\pi$ plasma levels have been proposed as useful to predict treatment efficacy or recurrence after surgery in oral-cavity cancer. Moreover, alterations of tumor-suppressor genes and/or oncogenes, which proved to be related to tumor aggressiveness in several tumor types, have been reported to affect sensitivity to drugs (Gottesman, 1994). Alterations of the TP53 tumor-suppressor gene (the most common genetic alterations found in human malignancies) were found to be a negative prognostic factor in different tumor types (Harris and Hollstein, 1993) and appeared to provide information on resistance or sensitivity to different chemical and physical agents (Righetti et al., 1996).

Resistance to chemotherapy has also been associated with decreased susceptibility to apoptosis (Reed, 1995), raising the possibility that cell-death determinants may influence treatment outcome. However, control of the apoptotic pathway is highly complex since it involves a multiplicity of growth-control factors, suggesting the combined consideration of its positive and negative regulators. In particular, among the different controlling genes, $b c l-2$ is known to be a negative regulator of cell death, whereas bax appears as a promoting factor with similar structure, able to form heterodimers with $b c l-2$ and thus counteract its death-repressor activity in several experimental models. The de-regulated expression of these genes in neoplastic tissues may confer resistance to chemotherapy by enabling cells to avoid apoptosis. Another tumor feature influencing clinical response to treatment is cell-proliferation rate, defined by quantification of S-phase cells (thymidine- or bromodeoxyuridine-labeling index, flow-cytometric $\mathrm{S}$ fraction) or of the whole cell-cycling fraction (Ki67, AgNORs) (Quinn and Wright, 1990). In fact, in vitro and in vivo experimental data indicate a direct relation between cell proliferation and response to chemotherapy or radiotherapy, and evidence in this regard has consistently emerged from retrospective and prospective studies in human tumors (Corvò et al., 1997; Silvestrini et al., 1998).

In the present study, we proposed to define the predictive role in terms of pathological response and the prognostic role in terms of

Grant sponsor: Italian National Research Council; Grant numbers: 96.00747.PF39; 96.00715.PF39; Grant sponsor: Associazione Italiana Ricerco Cancro.

*Correspondence to: Oncologia Sperimentale C, Istituto Nazionale Tumori, Via Venezian, 1, 20133 Milan, Italy. Fax: (39)2-2364366.

E-mail: acosta@istitutotumori.mi.it

Received 15 April 1998; Revised 8 June 1998 
disease-free survival (DFS) of GST- $\pi, \mathrm{p} 53$, bcl-2 and bax, immunohistochemically detected, and of S-phase cell fraction, as defined by thymidine labeling index (TLI) (Silvestrini et al., 1991), within a randomized phase III clinical trial on oral-cavity cancer, including surgery or primary chemotherapy.

\section{MATERIAL AND METHODS}

\section{Patient population}

Biological determinations were performed on tumors obtained from a series of 100 previously untreated patients, with resectable $\mathrm{T} 2(>3 \mathrm{~cm})-4 \mathrm{~N} 0-2 \mathrm{M} 0$ squamous-cell carcinoma of the oral cavity. Cases were among the series of 197 patients who entered a still open multicenter randomized trial started in 1989 in order to establish the role of PCT in patients with advanced resectable oral-cavity cancer (Grandi et al., 1994). In particular, the biological characterization was prospectively planned and carried out on tumors from all patients enrolled at the Istituto Nazionale Tumori of Milan from November 1989 to December 1997. Patients were less than 70 years old and randomly assigned to receive surgery (group A) or PCT followed by surgery (group B). PCT consisted of 3 cycles of cisplatin $\left(100 \mathrm{mg} / \mathrm{m}^{2}\right)$ plus 5 -fluorouracil $\left(1,000 \mathrm{mg} / \mathrm{m}^{2}\right)$ on days 1 to 5 as a 120-hr infusion repeated every 21 days. Surgery was planned within 3 weeks from the last chemotherapy cycle. Only high-risk patients (defined as those with positive surgical margins, extracapsular tumor spread and/or more than 3 node metastases) of both groups received $50 \mathrm{~Gy}$ of post-operative radiotherapy.

In group $\mathrm{B}$, patients progressive after the first cycle were immediately operated on. Surgery was performed in patients with stable disease after 2 cycles. Responsive patients, as defined by the WHO criteria, were operated on after having completed 3 cycles of PCT. Resected specimens were analyzed, and chemotherapyinduced necrosis was evaluated according to a 3-grade scale. Grade I was classified as the presence of gross residual tumor, grade II as the presence of isolated microscopic residual tumor foci and grade III as no detectable viable tumor, this last being defined as pathological complete remission. Pathological response was defined as the achievement of grade II regression (microresidual disease) and grade III (pathological complete remission).

Biological determinations were performed on biopsy specimens before PCT for patients in group B and on surgical specimens for patients in group A. For the determination of bax expression, tumor samples were available for 92 of the 100 patients. For the series of tumors for which biological information was available, clinical features, according to the TNM classification system (UICC, 1987), were similar to those of the overall series of patients entered in the randomized study (Table I).

In vitro determinations

Immediately after removal, biopsy or surgical tumor specimens were incubated with ${ }^{3} \mathrm{H}$-thymidine (Silvestrini kit; Euroframe, Asti,

\begin{tabular}{|c|c|c|c|c|}
\hline & \multicolumn{2}{|c|}{ Group $\mathrm{A}^{1}$} & \multicolumn{2}{|c|}{ Group B ${ }^{2}$} \\
\hline & $\begin{array}{c}\text { Overall } \\
\text { series } \\
(\mathrm{n}=99)\end{array}$ & $\begin{array}{c}\text { Biologically } \\
\text { characterized } \\
(\mathrm{n}=51)\end{array}$ & $\begin{array}{c}\text { Overall } \\
\text { series } \\
(\mathrm{n}=98)\end{array}$ & $\begin{array}{c}\text { Biologically } \\
\text { characterized } \\
(\mathrm{n}=49)\end{array}$ \\
\hline \multicolumn{5}{|c|}{ Initial tumor size } \\
\hline $\mathrm{T} 2(>3 \mathrm{~cm})$ & $43 \%$ & $45 \%$ & $51 \%$ & $43 \%$ \\
\hline $\mathrm{T} 3$ & $38 \%$ & $40 \%$ & $35 \%$ & $47 \%$ \\
\hline $\mathrm{T} 4$ & $19 \%$ & $15 \%$ & $14 \%$ & $10 \%$ \\
\hline \multicolumn{5}{|c|}{ Initial nodal status } \\
\hline No & $56 \%$ & $55 \%$ & $58 \%$ & $57 \%$ \\
\hline N1 & $27 \%$ & $31 \%$ & $27 \%$ & $26 \%$ \\
\hline $\mathrm{N} 2 \mathrm{a}$ & $6 \%$ & $4 \%$ & $5 \%$ & $6 \%$ \\
\hline $\mathrm{N} 2 \mathrm{~b}$ & $9 \%$ & $8 \%$ & $8 \%$ & $11 \%$ \\
\hline $\mathrm{N} 2 \mathrm{c}$ & $2 \%$ & $2 \%$ & $2 \%$ & - \\
\hline
\end{tabular}

${ }^{1}$ Surgery \pm RT. $-{ }^{2}$ Chemotherapy + surgery \pm RT.
Italy) and then processed for conventional histological procedures for determination of TLI, p53, bcl-2, bax and GST- $\pi$ expression. Investigators from Oncologia Sperimentale $\mathrm{C}$ of the National Cancer Institute of Milan have actively participated in national quality control programs activated in 1989 for TLI (Silvestrini et al., 1991) and for the other investigated biological markers. For all of the biomarkers under investigation, 2 investigators independently evaluated the specimens and inter-observer variability was always less than $10 \%$.

\section{TLI determination}

TLI was determined by autoradiography as described (Silvestrini et al., 1991) and evaluated independently by 2 observers by scoring a total of more than 3,000 tumor cells on different areas from the same tumor specimen. TLI was defined as the percentage ratio between labeled cells and total number of tumor cells.

\section{Immuno-histochemical determinations}

p53 expression. Histological sections $(4 \mu \mathrm{m})$ were incubated for $1 \mathrm{hr}$ with the PAb1801 monoclonal antibody (1:50 dilution; Oncogene Science, Uniondale, NY), which was raised against human 553 protein and recognizes wild-type and mutant forms of p53 protein. Specimens were then incubated with a goat anti-mouse immunoglobulin and processed with an avidin-biotin complex peroxidase method (Vectastain ABC kit; Vector, Burlingame, CA).

bcl-2 expression. Histological sections $(4 \mu \mathrm{m})$ were incubated for $1 \mathrm{hr}$ at room temperature with a mouse monoclonal anti-human bcl-2 oncoprotein (1:40 dilution; clone 124; Dakopatts, Copenhagen, Denmark). Specimens were then incubated with a goat anti-mouse immunoglobulin and treated with an avidin-biotin complex peroxidase method (Vectastain $\mathrm{ABC}$ kit, Vector).

For the short-fixation time $(6 \mathrm{hr})$, incubation of slides in a microwave oven was not necessary to retrieve antigen expression (Silvestrini et al., 1995). The fraction of p53- and bcl-2-positive tumor cells was evaluated independently by 2 observers by scoring a total of 1,000 to 3,000 tumor cells. Immunostaining for p53 and bcl-2 (at nuclear level for the former and cytoplasmic for the latter) was defined as the percentage ratio between positive and total number of tumor cells. Samples were considered positive if unequivocal brown staining was seen.

bax expression. Histological sections $(4 \mu \mathrm{m})$ were incubated for $2 \mathrm{hr}$ at $4^{\circ} \mathrm{C}$ with a rabbit polyclonal antibody $(1: 400$ dilution, clone N-20; Santa Cruz Biotechnology, Santa Cruz, CA). Specimens were then processed by using the Dako quick-staining labeled alkaline-phosphatase kit (Dako Chemate, Dakopatts).

GST- $\pi$ expression. Histological sections $(4 \mu \mathrm{m})$ were incubated with a rabbit polyclonal antibody (1:50 dilution; Ylem, Avezzano, Italy) for $2 \mathrm{hr}$ at $4^{\circ} \mathrm{C}$. Specimens were then incubated with a goat anti-rabbit immunoglobulin (Vector) and processed by using the Dako quick-staining labeled alkaline-phosphatase kit (Dako Chemate, Dakopatts).

The bax and GST- $\pi$ immunostaining was defined according to a qualitative scale. Tumor samples were considered negative in the absence of any cytoplasmic immunoreactivity or when weakly stained and positive when unequivocally stained.

Head-and-neck squamous-cell carcinomas with high p53, bcl-2, bax or GST- $\pi$ immunoreactivity were used as positive controls, while negative controls were obtained by omission of the specific primary antibody.

\section{Statistical analysis}

Spearman's rank-correlation coefficient was used to assess the association between TLI, p53 and bcl-2 expression considered as continuous variables. Wilcoxon's rank-sum test and Fisher's exact test were used to assess the relation between bax or GST- $\pi$, qualitatively graded, and TLI, p53 and bcl-2 expression.

In group $\mathrm{B}$, the relation between pre-treatment values of biological variables and pathological complete remission and grade II pathological response was assessed by the $\chi^{2}$ test. DFS was 
computed in both groups, starting from the date of surgery, by the Kaplan-Meier product-limit method. The Cox model was used to assess differences among sub-groups. Since patient accrual is ongoing, the present is an interim analysis based on available data.

For clinical correlations, biomarkers were used as categorical variables. For TLI, the cut-off value of $14 \%$ (which represents the median value for the present series) was used. p53, bcl-2, bax and GST- $\pi$ expression were categorized as negative $v s$. positive.

\section{RESULTS}

Immunoreactivity to p53 protein, characterized by nuclear staining, was detected in $52 \%$ of cases. The number of p53-positive cells in individual tumors ranged from $1 \%$ to $86 \%$, with a median value of $29 \%$. Positivity to bcl-2, bax and GST- $\pi$ proteins was localized in the cytoplasm of tumor cells, and the frequency of positive tumors was $15 \%, 55 \%$ and $57 \%$, respectively. TLI values showed a log-normal distribution, ranging from $0.01 \%$ to $32.6 \%$ for the different tumors, with a median value of $14 \%$. Tumor biological characteristics were similar for patients entered in the 2 groups of the clinical study (Table II).

The only relation found among the biomarkers was an inverse one between TLI and GST- $\pi$ expression and a trend in favor of a direct relation between bcl-2 and bax expression $(p=0.03)$. In particular, the median TLI value was significantly higher in GST- $\pi$-negative than in GST- $\pi$-positive tumors ( $16.6 \% v s .12 .8 \%$, respectively; $p=0.01$ ), whereas 9 of 11 bcl-2-positive tumors also expressed bax.

\section{Biomarkers as prognostic or predictive variables}

In light of the clinical protocol, we analyzed the relevance of biological variables as prognostic indicators in terms of DFS in patients treated with surgery alone (group A) and as predictors of pathological response and DFS in patients treated with PCT and surgery (group B). The median follow-up was 37 months (range, 1 to 90 months). At this writing, 31 patients have relapsed: 16 at local-regional sites, 3 at distant sites and 12 at a regional level. The overall clinical outcome of patients with available biological information was similar to that of all enrolled patients (data not shown).

In patients treated with surgery alone, neither bcl-2/bax expression nor TLI provided information on long-term clinical outcome (Table III), and moderate/strong GST- $\pi$ and lack of $\mathrm{p} 53$ protein expression were favorable, though not statistically significant, indicators of DFS.

Pathological response was obtained in 18 patients treated with PCT: 9 grade III pathologically complete remissions and 9 grade II pathological regressions. Pathological response rates and DFS (Table IV) were independent of TLI and p53 expression. Conversely, the lack of GST- $\pi$ and bax expression was related to pathological response rates 3 - and 2-fold higher, respectively, than

\begin{tabular}{|c|c|c|}
\hline & Group $A^{1}$ & Group B ${ }^{2}$ \\
\hline \multicolumn{3}{|l|}{ p53 expression } \\
\hline Positive cases & $55 \%$ & $49 \%$ \\
\hline 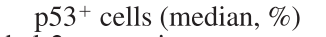 & 34.5 & 24.6 \\
\hline \multicolumn{3}{|l|}{ bcl-2 expression } \\
\hline Positive cases & $13 \%$ & $16 \%$ \\
\hline bcl- $2^{+}$cells (median, \%) & 5.0 & 12.5 \\
\hline \multicolumn{3}{|l|}{ bax expression } \\
\hline Positive cases & $55 \%$ & $56 \%$ \\
\hline \multicolumn{3}{|l|}{ GST- $\pi$ expression } \\
\hline Positive cases & $62 \%$ & $53 \%$ \\
\hline \multicolumn{3}{|l|}{ TLI } \\
\hline Median value & $11.9 \%$ & $14.5 \%$ \\
\hline
\end{tabular}

${ }^{1}$ Surgery \pm RT. $-{ }^{2}$ Chemotherapy + surgery \pm RT.
TABLE III - BIOMARKERS AND CLINICAL OUTCOME IN PATIENTS SUBMITTED TO SURGERY AS FIRST-LINE TREATMENT (GROUP A)

\begin{tabular}{ccc}
\hline & $\begin{array}{c}\text { Disease-free } \\
\text { survival at 3 years, } \%^{1}\end{array}$ & HR $(95 \% \mathrm{CI})^{2}$ \\
\hline $\begin{array}{l}\text { GST- } \pi \text { expression } \\
\text { Negative/weak }\end{array}$ & $51(32)$ & $1.5(0.5-4.2)$ \\
$\begin{array}{l}\text { Moderate/strong } \\
\text { p53 expression }\end{array}$ & $71(19)$ & \\
$\quad \begin{array}{l}\text { Negative } \\
\text { Positive }\end{array}$ & $76(24)$ & \\
bcl-2 expression & $52(27)$ & $2.0(0.7-5.8)$ \\
$\quad$ Negative & $61(44)$ & $1.2(0.3-5.3)$ \\
Positive & $71(7)$ & \\
bax expression & $67(21)$ & $1.2(0.4-3.4)$ \\
$\quad$ Negative & $58(26)$ & $1.5(0.6-4.2)$ \\
Positive & $62(31)$ & \\
TLI & $64(20)$ & \\
$\quad \leq 14 \%$ &
\end{tabular}

${ }^{1}$ In parentheses, number of cases.- ${ }^{2} \mathrm{HR}$, hazard ratio; CI, confidence interval.

TABLE IV - BIOMARKERS AND CLINICAL OUTCOME IN PATIENTS SUBMITTED TO PRIMARY CHEMOTHERAPY (GROUP B)

\begin{tabular}{cccc}
\hline & $\begin{array}{c}\text { Pathological } \\
\text { response }^{1}, \%^{2}\end{array}$ & $\begin{array}{c}\text { Disease-free } \\
\text { survival at } \\
3 \text { years, } \%^{2}\end{array}$ & HR $(95 \% \mathrm{CI})^{3}$ \\
\hline $\begin{array}{c}\text { GST- } \pi \text { expression } \\
\quad \text { Negative/weak }\end{array}$ & $50(15 / 30)$ & $72(30)$ & \\
$\begin{array}{l}\text { Moderate/strong } \\
\text { p53 expression }\end{array}$ & $16(3 / 19)$ & $67(19)$ & $1.2(0.4-3.3)$ \\
$\quad \begin{array}{l}\text { Negative } \\
\text { Positive }\end{array}$ & $40(10 / 25)$ & $67(25)$ & $1.3(0.5-3.8)$ \\
bcl-2 expression & $33(8 / 24)$ & $74(24)$ & \\
$\quad \begin{array}{l}\text { Negative } \\
\text { Positive }\end{array}$ & $37(15 / 41)$ & $75(41)$ & \\
bax expression & $38(3 / 8)$ & $47(8)$ & $2.8(0.9-8.9)$ \\
$\quad \begin{array}{l}\text { Negative } \\
\text { Positive }\end{array}$ & $50(10 / 20)$ & $68(20)$ & $1.1(0.4-3.4)$ \\
TLI & $28(7 / 25)$ & $71(25)$ & \\
$\quad \leq 14 \%$ & $33(7 / 21)$ & $70(21)$ & $1.0(0.4-3.0)$ \\
$\quad>14 \%$ & $39(11 / 28)$ & $70(28)$ & \\
\hline
\end{tabular}

${ }^{1}$ Grade-II and Grade-III regression.-- ${ }^{2}$ In parentheses, number of cases. $-{ }^{3} \mathrm{HR}$, hazard ratio; CI, confidence interval.

those observed for patients with tumors over-expressing GST- $\pi$ and bax. However, both variables lost relevance as predictors of DFS. Patients with bcl-2-negative tumors had a $75 \%$ probability of DFS compared with only $47 \%$ observed for patients with bcl-2expressing tumors $(p=0.07)$.

Since all responder patients were alive and disease-free at 3 years, we analyzed DFS as a function of biological variables within the sub-set of non-responder patients. Again, bcl-2 expression was the only discriminant of DFS (Fig. 1). In particular, all patients with bcl-2-positive tumors ( 5 cases) relapsed within 1 year, whereas a $60 \%$ probability of DFS was observed for the 26 patients with bcl-2-negative tumors $(p=0.02)$.

\section{DISCUSSION}

Correlative studies, in which the determination of biological markers is foreseen and prospectively performed within the context of clinical treatment protocols, are of remarkable interest to validate preliminary evidence of some relation between biomarkers and clinical outcome and to verify the usefulness of biological characterization in clinical practice. In fact, for head-and-neck cancers, the role of biomarkers on clinical outcome has been generally derived from retrospective analyses on different sub-sets of patients and rarely prospectively validated, with possible selection bias for case series under investigation, as well as 


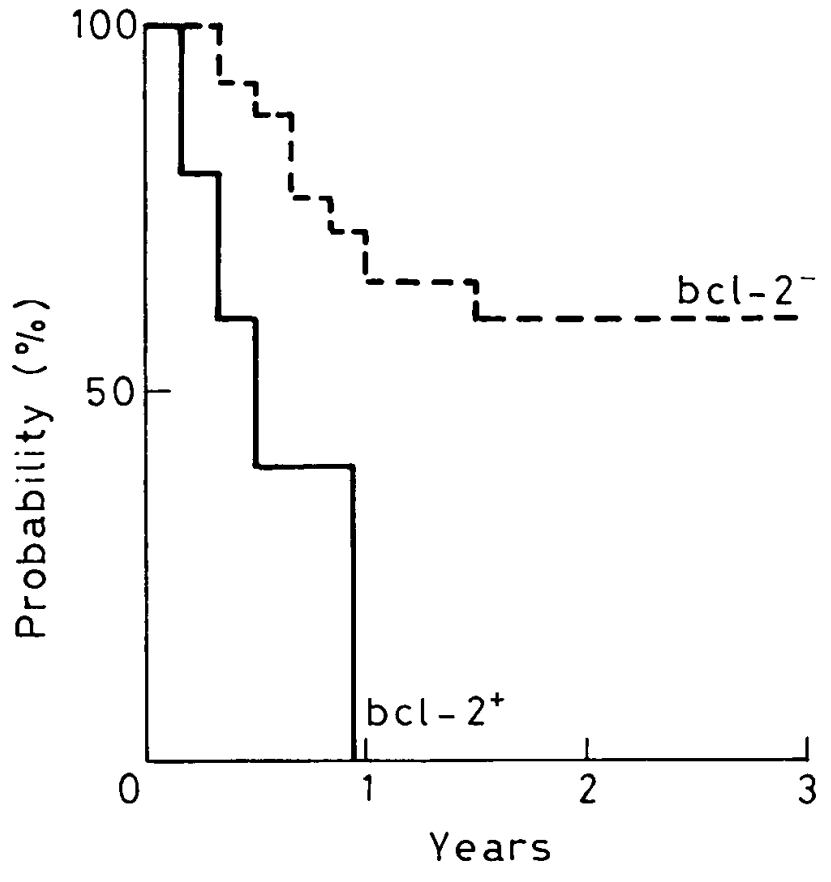

FIGURE 1 - Disease-free survival as a function of bcl-2 expression in non-responder patients. ---, bcl-2-negative tumors; - - bcl-2-positive tumors. Hazard ratio $=4.3(95 \%$ confidence interval $=1.3-14.3), p=$ 0.02 .

possible different predictivity of biomarkers in different tumor sites, even in consideration of clinical and biological heterogeneity of this neoplasm. Moreover, the different functional aspects of tumor cells have been occasionally investigated within a multifactorial frame, to assess their relative role in predicting treatment response. The activation of randomized clinical protocols on neo-adjuvant chemotherapy, in which pathological response to chemical agents is assessed after a few cycles, and the high feasibility of immuno-cytochemical determination of multiple markers on small tumor specimens prompted correlative studies on consecutive series of patients.

In the present study, we investigated the prognostic and predictive role of biomarkers involved in different cell functions, such as proliferation (TLI), repair of DNA damage (p53 expression), control of programmed cell death (bcl-2 and bax expression) and detoxification of electrophilic compounds (GST- $\pi$ expression), on clinical outcome of patients with resectable oral-cavity cancer and entered in a randomized trial comparing surgery alone with primary chemotherapy plus surgery. A main purpose of the study was to evaluate the predictive role of pre-treatment biomarkers in terms of obtaining a pathological response, with a view to the clinical need to look at biomarkers able to indicate highly chemosensitive patients who may really benefit from PCT. Although the biological characterization was prospectively carried out on tumor specimens obtained only at the Istituto Nazionale Tumori of Milan (accounting for about $50 \%$ of the whole enrolled series), results could be considered as representative of those achievable from a multicenter study. In fact, the main clinico-pathological features of the patient series biologically characterized were superimposable to those observed for the overall series.

In the present series of resectable T2-4N0-2 squamous-cell carcinomas of the oral cavity, the fraction of tumors rapidly proliferating and over-expressing p53, bcl-2, bax or GST- $\pi$ was in keeping with published results on this tumor type.

As a whole, in the group subjected to surgery alone with or without radiotherapy, none of the investigated markers significantly influenced clinical outcome. The findings regarding expres- sion of p53, GST- $\pi$ or bcl-2 were in agreement with results on series of patients similar in terms of site and stage of disease (Veneroni et al., 1997). The lack of prognostic relevance of cell proliferation, as reported earlier, could in part be explained by the impact of radiation therapy, which was administered in about $40 \%$ of cases. In fact, a radiation benefit for sub-sets of patients with tumors characterized by specific biological features or similar molecular pathways has been reported (Silvestrini et al., 1997).

Conversely, the investigated markers variously predicted chemotherapeutic efficacy and outcome. In particular, patients with GST- $\pi$-negative tumors more frequently achieved pathological response to cisplatin and 5-fluorouracil treatment than patients with GST- $\pi$-positive tumors. Similar findings have been observed in patients with non-small-cell lung cancer submitted to a similar chemotherapeutic regimen (Bai et al., 1996) as well as in patients with pharyngeal and laryngeal carcinoma treated with radiotherapy (Tanita et al., 1993). bcl-2 expression was not indicative of pathological response, in agreement with results reported for patients with locally advanced squamous-cell carcinoma of the esophagus (Puglisi et al., 1996) or with epithelial ovarian tumors (van der Zee et al., 1995; Herod et al., 1996) treated with chemotherapeutic regimens including cisplatinum. However, bcl-2 expression appeared to be a significant predictor of 3-year DFS. Such a finding indicates that absence of bcl- 2 expression, by itself an unfavorable prognostic marker in several neoplasms submitted to local-regional treatment, becomes a favorable indicator of long-term outcome in non-responder patients, i.e., in a situation of natural history. A similar observation was reported by Gallo et al. (1996) and Friedman et al. (1997) in patients with early-stage head-and-neck cancer treated with curative radiotherapy or surgery. The mechanisms by which disordered bcl-2 expression could lead to shorter survival are at present speculative. In fact, in addition to the possibility that absent or weak bcl-2 expression favors apoptosis induced by drugs, the maximum influence of bcl-2 in nonresponder patients could be explained by the hypothesis that bcl-2 over-expression prevents natural apoptosis in such tumors, thereby inducing more rapid accumulation of malignant cells. Although in other neoplasms the determination of other apoptosis-related markers allowed more accurate detection of patients with an unfavorable clinical outcome, in the present study, the combined analysis of bcl- 2 and bax only marginally improved bcl-2 predictivity. In fact, lack of bax expression provided prognostic information only in non-responder patients with bcl-2-negative tumors (data not shown).

No relation was observed between $\mathrm{p} 53$ expression and chemotherapy response, in agreement with some data reported in esophageal (Puglisi et al., 1996) and ovarian (van der Zee et al., 1995; Herod et al., 1996) carcinomas but not with other findings on the latter tumor type (Righetti et al., 1996). Such an observation emphasizes that resistance to regimens including cisplatin is a complex process, involving defects in cell-membrane transport, detoxification and alternative metabolic pathways or changes in DNA repair. Cell proliferation did not appear as a predictive factor in patients submitted to primary chemotherapy. However, the presence of an S phase-specific drug, i.e., 5-fluorouracil, in the chemotherapeutic regimen could be responsible for a possible advantage derived from primary chemotherapy for rapidly proliferating tumors, whose clinical behavior paralleled that observed for more indolent, slowly proliferating tumors.

In conclusion, this interim analysis indicates that some biofunctional markers, which appear as prognostic indicators in several tumor types, can provide information on pathological response to primary chemotherapy and may help us to understand treatment efficacy at a cellular level. Obviously, suggestions emerging from this correlative study should be interpreted with caution and need to be confirmed within this ongoing clinical trial, as well as validated in other and similar clinical settings.

\section{ACKNOWLEDGEMENTS}

We thank Mrs. B. Canova and Mrs. B. Johnston for editorial assistance. 


\section{REFERENCES}

Bai, F, NaKanishi, Y, Kawasaki, M. Takayama, K, Yatsunami, J, Hai PeI, X., Tsuruta, N., WaKamatsu, K. and Hara, N., Immunohistochemical expression of glutathione $S$-transferase- $\pi$ can predict chemotherapy response in patients with non-small cell lung carcinoma. Cancer, 78, 416-421 (1996)

Browman, G.P., Evidence-based recommendations against neoadjuvan chemotherapy for routine management of patients with squamous cell head and neck cancer. Cancer Invest., 12, 662-671 (1994).

Corvò, R., Giaretti, W., Sanguineti, G., Geido, E., Bacigalupo, A., Orecchia, R., Benasso, M., Numico, G.M., Merlano, M., Margarino, G. and VitALE, V., Chemoradiotherapy as an alternative to radiotherapy alone in fast proliferating head and neck squamous cell carcinomas. Clin. Cancer Res., 3, 1993-1997 (1997).

Friedman, M., Grey, P., Venkatesan, T.K., Bloch, I., Chawla, P. CALDARELli, D.D and CoON, J.S., Prognostic significance of bcl-2 expression in localized squamous cell carcinoma of the head and neck. Ann. Otol. Rhinol. Laryngol., 106, 445-450 (1997).

Gallo, O., Boddi, V., Calzolari, A Simonetti, L. Trovati, M and BIANCHI, S., bcl-2 protein expression correlates with recurrence and survival in early stage head and neck cancer treated by radiotherapy. Clin Cancer Res., 2, 261-267 (1996)

GotTESMAN, M.M., Report of a meeting: molecular basis of cancer therapy J. nat. Cancer Inst., 86, 1277-1285 (1994)

GRANDI, C., and 12 OTHERS, Surgery versus primary chemotherapy and surgery in cancer of the oral cavity: interim report of a randomized study. Fourth Research Workshop on the Biology, Prevention, and Treatment of Head and Neck Cancer. Head Neck, 16, 482 (1994).

Harris, C.C. and Hollstein, M., Clinical implications of the p53 tumor-suppressor gene. N. Engl. J. Med., 329, 1318-1327 (1993).

Herod, J.J.O., Eliopoulos, A.G., Warwick, J., Niedobitek, G., Young, L.S. and KERR, D.J., The prognostic significance of bcl-2 and p53 expression in ovarian carcinoma. Cancer Res., 56, 2178-2184 (1996).

Okuyama, T., Maehara, Y., Endo, K., Baba, H., Adachi, Y., Kuwano, K. and SugIMACHI, K., Expression of glutathione $S$-transferase- $\pi$ and sensitivity of human gastric cancer cells to cisplatin. Cancer, 74, 1230-1236 (1994).

Puglisi, F., Di Loreto, C., Panizzo, R., Avellini, C., Fongione, S., CACITTI, V. and Beltrami, C.A., Expression of p53 and bcl-2 and response to preoperative chemotherapy and radiotherapy for locally advanced squamous cell carcinoma of the oesophagus. J. clin. Pathol., 49, 456-459 (1996).

QuinN, C.M. and WRIGHT, N.A., The clinical assessment of proliferation and growth in human tumours: evaluation of methods and applications as prognostic variables. J. Pathol., 160, 93-102 (1990)

REED, J.C., Regulation of apoptosis by bcl-2 family proteins and its role in cancer and chemoresistance. Curr. Opin. Oncol., 7, 541-546 (1995).

RigHeTtI, S.C. and 13 otHERs, A comparative study of $p 53$ gene mutations, protein accumulation and response to cisplatin-based chemotherapy in advanced ovarian carcinoma. Cancer Res., 56, 689-693 (1996).

Silvestrini, R., Daidone, M.G., Veneroni, S., Benini, E., Scarfone, G., Zanaboni, F., Villa, A., Presti, M., Danese, S. and Bolis, G., The clinical predictivity of biomarkers of stage III-IV epithelial ovarian cancer in a prospective randomized treatment protocol. Cancer, 82, 159-167 (1998).

Silvestrini, R., Rao, S., Benini, E., Daidone, M.G. and Pilotti, S., Immunohistochemical detection of p53 in clinical breast cancers: a look at methodologic approaches [Letter]. J. nat. Cancer Inst., 87, 1020 (1995).

Silvestrini, R. and The SiCCAB Group for Quality Control of Cell Kinetic Determination, Feasibility and reproducibility of the $3 \mathrm{H}-\mathrm{TdR}$ labeling index in breast cancer. Cell Prolif., 21, 437-445 (1991)

Silvestrini, R., Veneroni, S., Benini, E., Daidone, M.G., Luisi, A., Leutner, M., Maucione, A., Kenda, R., Zucali, R. and Veronesi, U., Expression of p53, glutathione $S$-transferase- $\pi$, and bcl- 2 proteins and benefit from adjuvant radiotherapy in breast cancer. J. nat. Cancer Inst., 89, 639-645 (1997)

Tanita, J., Tsuchida, S., Hozawa, J. and Sato, K., Expression of glutathione $S$-transferase- $\pi$ in human squamous cell carcinomas of the pharynx and larynx. Cancer, 72, 569-576 (1993).

UICC (INTERNATIONAL UNION AGAINST CANCER), TNM classification of malignant tumors (4th ed.), Springer, Berlin (1987).

Van der Zee, A.G.J., Hollema, H., Suurmeijer, A.J.H., Krans, M., Sluiter, W.J., Willemse, P.H.B., Aalders, J.G. and De Vries, E.G., Value of P-glycoprotein, glutathione $S$-transferase pi, c-erbB-2, and p53 as prognostic factors in ovarian carcinomas. J. clin. Oncol., 13, 70-78 (1995). Veneroni, S., Silvestrini, R., Costa, A., Salvatori, P., Faranda, A. and MOLINARI, R., Biological indicators of survival in patients treated by surgery for squamous cell carcinoma of the oral cavity and oropharynx. Europ. J. Cancer Oral Oncol., 33, 408-413 (1997). 